\title{
Experimental Study on Boron Nitride Intumescent Fire Retardant Coating
}

\author{
Peng WANG \\ Chinese people's Armed Police Force Academy, Hebei Langfang, 065000, China \\ wangpengcapf@139.com
}

Keywords: Fire Retardant, Intumescent Coating, Boron Nitride, Borax, Melamine.

\begin{abstract}
Boron nitride intumescent fire retardant coating with borax and melamine as the main components was experimentally studied. The formulation of coating was designed by orthogonal experiment; fire resistance time was tested in the intumescent fire retardant coating testing furnace; the micro-structure of the coating was studied by SEM. Experimental results showed that: after heated, a layer of dark green, compact lazed $\mathrm{B}_{2} \mathrm{O}_{3}$ formed on the surface of intumescent coating; and a structure of porous spongy $\mathrm{BN}$ formed in the inner of coating. This structure makes the coating has a high resistance to heat. But because the melting point of borax is low, the coating can't withstand high temperature, it will soften at high temperatures. This has seriously affected the fire resistance time of the coating. The method for solving this problem is to find a new kind of nitrogen containing compounds, which makes the reaction of borax to boron nitride more easily. The experiments prove that, intumescent fire retardant coating with borax and nitrogen containing compound is feasible.
\end{abstract}

\section{Introduction}

Fire retardant coating for steel structure can form a heat insulation protection layer and can improve the fire resistance limit of steel structure in building [1]. For example, an intumescent flame-retardant coating was prepared by unsaturated polyester resin and epoxy resin as two-component matrix resins, ammonium polyphosphate (APP) as acid source, melamine (MEL) as the blowing agent and pentaerythritol (PER) as carbon agent, expandable graphite as synergistic agent, adding titanium dioxide $\left(\mathrm{TiO}_{2}\right)$, solvent and other assistants [2]. This kind of fire retardant coatings are P-C-N system fire retardant coatings.

In recent years, the progress of fire retardant coating is mainly focused on the improvement of the P-C-N system [3]. And new fire retardant coating systems have also been reported. Two experts from Tongji University studied a novel transparent intumescent fire resistant coating which was developed by the P-Si synergistic flame retardant and melamine formaldehyde resin. The P-Si synergistic flame retardant could improve the thermo-oxidation resistance of transparent fire resistant coating [4-7].

This paper studies the feasibility of intumescent fire retardant coating with borax and nitrogen containing compound. The intumescent mechanism of this novel fire retardant coating is completely different from that in the P-C-N system, the intumescent mechanism is mainly rely on the expansion of borax. 


\section{Experiment}

\section{Reagents}

The reagents in experiments are listed as follows: Borax (sodium tetraborate), AR, Tianjin Ding Sheng Xin Chemical Co., Ltd.; Aluminate cement (CA-50 A-700), technical pure, Zhengzhou Jianai Special Aluminate Co. Ltd.; Hydroxypropyl methyl cellulose (HMC), CP, Tianjin Kwangfu Fine Chemical Industry Research Institute; Melamine (cyanurotriamide/ trimeric cyanamide), CP, Hushi brand, Chinese Medicine Group Chemical Reagent Co., Ltd.

\section{Instruments}

The instrument used to test the fire resistance time of fire retardant coating is called "intumescent fire retardant coating testing furnace", invented by Beijing Institute of Aerial Materials China Aviation Industry Corporation [8]. Figure 1 shows the sections of the furnace. During testing, only one side of the specimens was painted with coating and another side remained uncoated. The uncoated sides of two specimens were attached at the lower end of a cooling chip respectively, which was made of steel or heat-resistant alloy. Wire was used to combine the cooling chip and the two samples together. The cooling chip and the two samples were placed on the bracket in the furnace vertically. One thermocouple was placed in the groove of the cooling chip to measure the back-side temperature of the specimens, and another thermocouple was placed in the furnace to monitor the temperature of the furnace. The time-temperature curve in the furnace is in accordance with the ISO 834 standard curve. The time when the back side temperature of the samples reaches $580^{\circ} \mathrm{C}$ is defined as the fire resistance time.
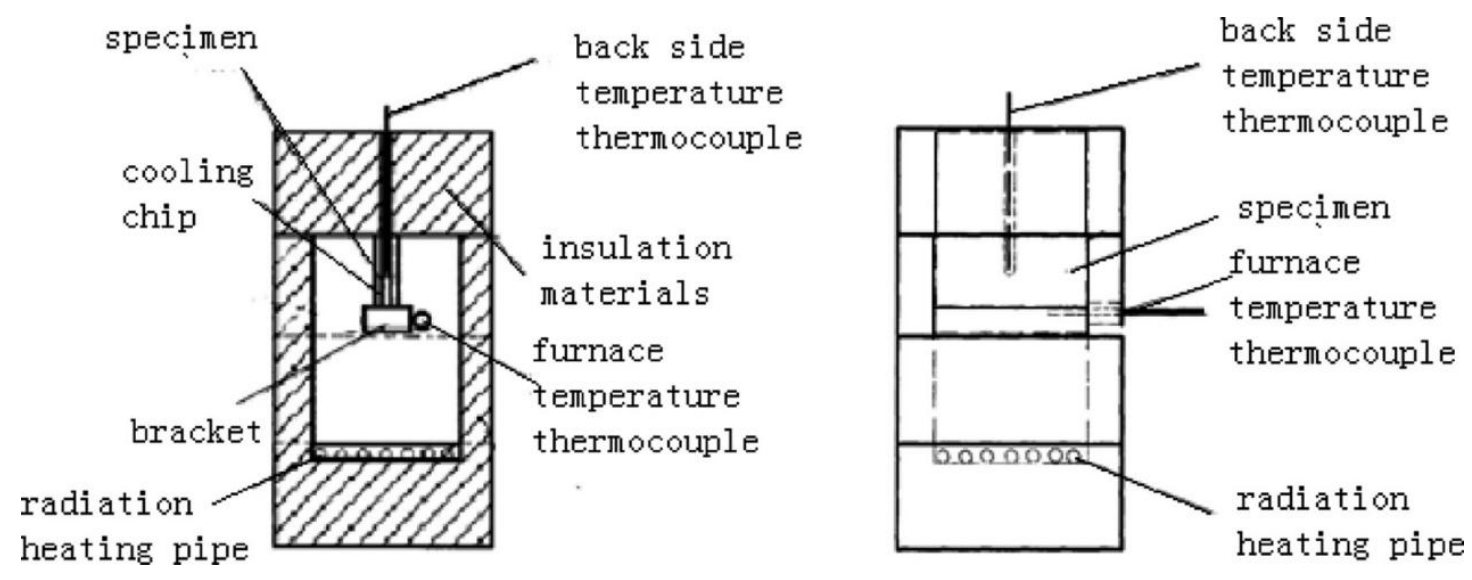

Fig. 1 Sections of the intumescent fire retardant coating testing furnace.

The scanning electron microscope used is TM3030Plus tabletop microscope, manufactured by Hitachi.

\section{Orthogonal Experimental Design}

The formulation of fire retardant coating is designed by orthogonal method, thus $\mathrm{L}_{9}\left(3^{4}\right)$ orthogonal table is chosen. The formulation of coating contains 4 reagents: borax, aluminate cement, melamine and HMC. The first factor in orthogonal experiment is borax whose function is expansion; the second factor is melamine whose function is reaction with borax to form $\mathrm{BN}$; the third factor is cement whose function is water hardening and to make powder form coating. Every factor has 3 levels, and the fourth factor is error. According to $\mathrm{L}_{9}\left(3^{4}\right)$ orthogonal table, there are 9 formulations, shown in table 1 . In addition, every formulation has $1 \mathrm{~g} \mathrm{HMC}$ as thickening agent. 


\section{Experimental Method}

Sample Preparation. According to the formulations in table 1, borax, melamine, aluminate cement and HMC were smashed and mixed in high speed disintegrator. The powder was mixed with some water, the mass ratio of powder to water was about $2 / 1$, so a thick mud like coating was got. The mud coating was painted on a specimen substrate with a brush. The specimen substrate was a Q235 steel plate of $80 \mathrm{~mm} \times 40 \mathrm{~mm} \times 1.2 \mathrm{~mm}$. Before brushing the coating, the steel substrates were cleaned thoroughly. The substrates were painted repeatedly, so that the coating on the substrates could have a certain thickness. After the coating was completely dry and solid, the surface of coating was rubbed down with sandpaper, until the surface was flat and the thickness was equal. The fire retardant coating is thin but not super thin, so the thickness should be $5 \mathrm{~mm}$ [1]. Because the thickness of substrate is $1.2 \mathrm{~mm}$, so the whole thickness of a specimen should be $6.2 \mathrm{~mm}$. At first, after repeatedly brushed, the thickness of specimens was more than $6.2 \mathrm{~mm}$. The thickness of a specimen was ground thin by sandpapers; at the same time measured by a vernier caliper until the thickness was $6.2 \mathrm{~mm}$. The prepared specimen is shown in Fig.2.
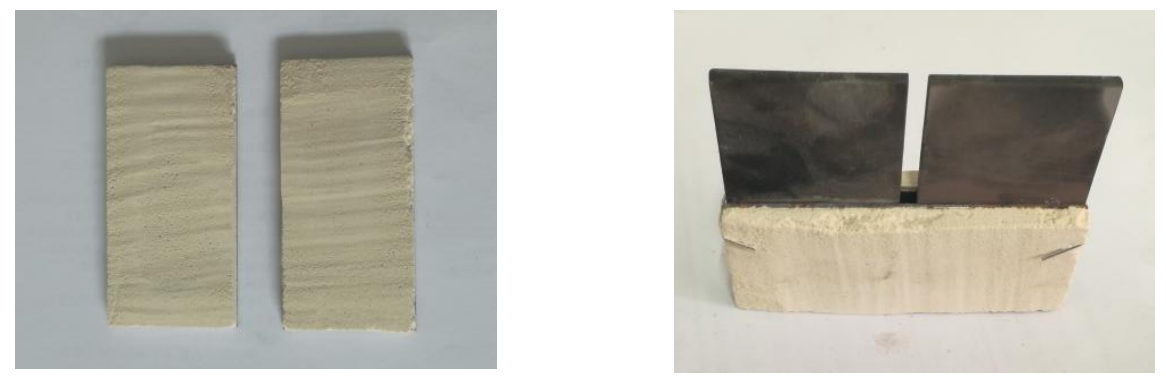

Fig. 2 Prepared specimen.

Experimentation. Fire resistance time of 9 formulations was tested in the furnace, and the time-temperature curve was recorded. The heated specimens were examined and took photographs. The results were analyzed of variance, and got the best formulation. According to GB/T 9978.1-2008 [9], the best formulation coating was heated to $945^{\circ} \mathrm{C}$ in a muffle furnace. The surface and internal structure of the heated coating were examined by scanning electron microscope (SEM). The SEM pictures were taken at 100 and 1000 magnifications.

\section{Result and Discussion}

\section{Fire resistance time}

The time-temperature curve of 9 formulations is shown in figure 3: the heavy line above indicates the standard heating curve [9]; and the fine lines below indicate the heating curve of 9 formulations. 


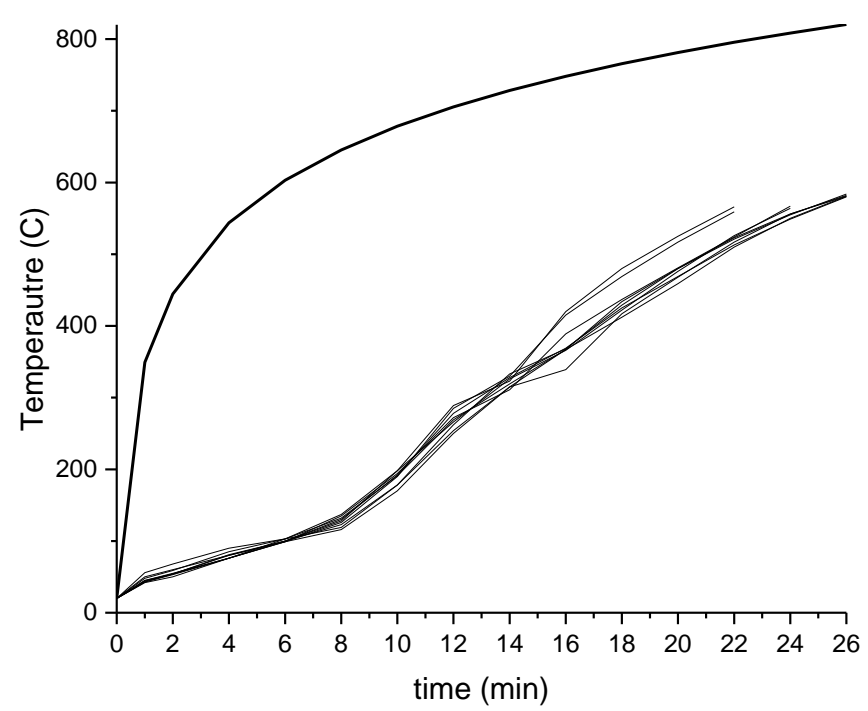

Fig.3 Time-temperature curves of 9 formulations.

The result data of fire resistance time of 9 formulations were got and the variance analysis as shown in table 1:

Table 1. The formulations, result data and analysis of orthogonal experiments.

\begin{tabular}{|l|l|l|l|l|l|}
\hline formulation & borax/g & melamine/g & cement/g & error & fire resistance time/s \\
\hline 1 & 70 & 15 & 10 & & 1633 \\
\hline 2 & 70 & 20 & 15 & & 1897 \\
\hline 3 & 70 & 25 & 20 & & 1828 \\
\hline 5 & 80 & 15 & 15 & & 1854 \\
\hline 6 & 80 & 20 & 20 & & 1866 \\
\hline 7 & 80 & 25 & 10 & & 1837 \\
\hline 8 & 90 & 15 & 20 & & 1770 \\
\hline 9 & 90 & 20 & 10 & & 1659 \\
\hline mean value 1 & 1786 & 1752 & 1710 & 1767 & \\
\hline mean value 2 & 1852 & 1807 & 1851 & 1835 & \\
\hline mean value 3 & 1744 & 1823 & 1821 & 1780 & \\
\hline range & 108 & 70 & 142 & 67 & \\
\hline SSD & 17900 & 8207 & 33439 & 7655 & \\
\hline F-rate & 2.338 & 1.072 & 4.368 & 1.000 & \\
\hline significance level & no & no & no & & \\
\hline
\end{tabular}

As shown in table 1: the sum of square of deviation (SSD) caused by error is the smallest, and all the SSD of other factors are bigger than that of error, so the experimental data are valid. The formulation of aluminate cement $15 \mathrm{~g}$, borax $80 \mathrm{~g}$, melamine $25 \mathrm{~g}$, and HMC $1 \mathrm{~g}$ is the best. All the fire resistance time of the 9 formulations are about $30 \pm 2 \mathrm{~min}$, and all factors have no significant effect on the experimental results. The results are much less than in the requirement of GB14907 [1]. 
Thus the fire resistance time can't be improved by changing the formula ratio; but can only be improved by changing the ingredients in formulation.

\section{Examination of Heated Coating}

Through the observation of the heated specimens, it is found that:

(a) The specimens are intumescent, and the intumescent specimens are hard and brittle.

(b) The surface of intumescent coating is covered by a layer of dark green, compact glaze while the internal structure is loose, porous sponginess. The structures of intumescent coating are significantly different from inside to outside, as shown in figure.4:

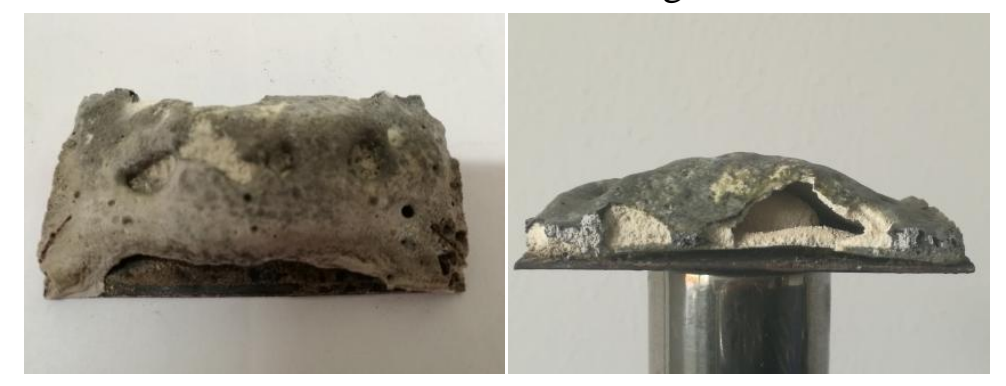

Fig.4 The heated coating is intumescent.

(c) The specimens are break away from substrates and a big interspace between the intumescent coating and the substrate is formed accordingly. The specimens are soften, which make the coating flow in the direction of gravity, as shown in figure.5:

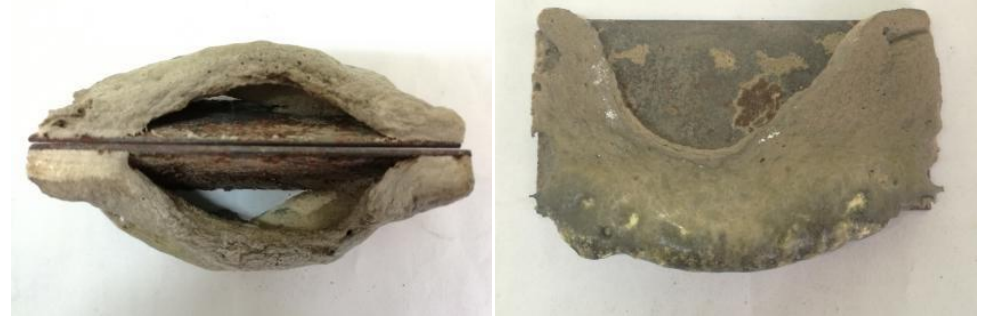

Fig. 5 The interspace between the intumescent coating from the substrate and flow in the direction of gravity.

Comprehensive analysis of the above phenomena indicates the reason why the fire resistance time is $30 \mathrm{~min}$. That is because the coating will melt at the temperature of less than $800^{\circ} \mathrm{C}$. The fusion of coating makes the separation from substrate and the flow in the direction of gravity. That causes the coating to lose the heat insulation ability. This temperature is about the melting point of borax $\left(741^{\circ} \mathrm{C}\right)$. This is the reason why the formulation contains melamine. Because the melamine (C3H6N6) is a nitrogen containing compound that can react with borax to form BN whose melting point is very high $\left(3000^{\circ} \mathrm{C}\right)$. The formed $\mathrm{BN}$ is the porous sponginess inside the intumescent coating, which formed the main body of the intumescent coating. If there were no melamine only borax in the formulation, then the expansion rate of the intumescent coating will be bigger, but the coating can't withstand high temperature of $945^{\circ} \mathrm{C}$ as required. Borax will melt at the temperature of $741^{\circ} \mathrm{C}$, and become compact glaze of $\mathrm{B}_{2} \mathrm{O}_{3}$ after cooling. Because of the thickness of $5 \mathrm{~mm}$, the inner section can't contact with oxygen, so the porous $\mathrm{BN}$ is formed; the surface can contact with oxygen, so the compact $\mathrm{B}_{2} \mathrm{O}_{3}$ is formed. That's why the surface of intumescent coating is compact glaze but the internal structure is porous sponginess. Although melamine's added, the melting of 
borax is still very obvious, as shown in picture 3. How to solve this problem is the key technology of B-N intumescent fire retardant coating. The fire resistance time can't be improved by increasing melamine as analyzed in table 1, thus other nitrogen containing compounds that is more easily to react with borax to form $\mathrm{BN}$ should be researched.

\section{Observation of Micro-structure}

In order to further study the structure of the intumescent coating, both the compact $\mathrm{B}_{2} \mathrm{O}_{3}$ layer on the surface and porous BN structure in the inner of the intumescent coating was observed by SEM, as shown in figure. 6 . This structure makes the coating has a high resistance to heat. The compact glazed surface prevents the hot air from entering, and protects the cold air in the internal structure. The porous sponginess in the internal structure has a very small thermal conductivity to hinder the heat transfer.

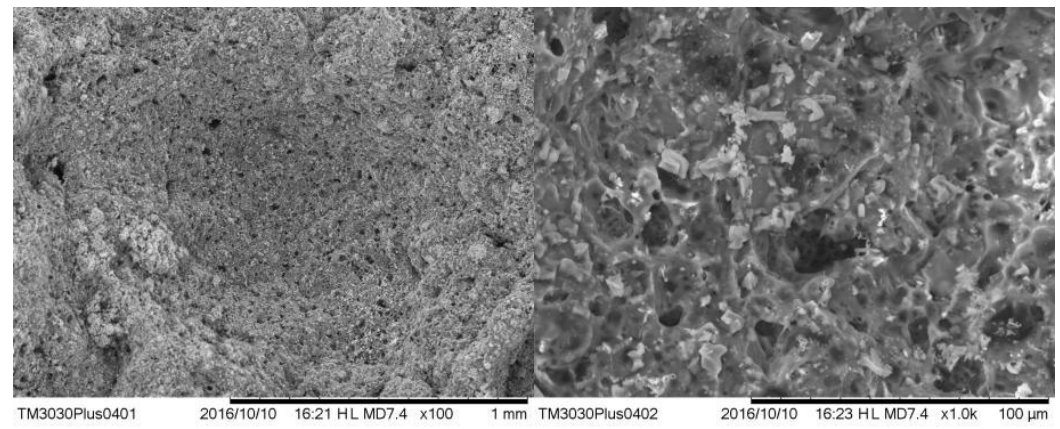

(a) $\times 100$

(b) $\times 1000$

Fig.6 .1 The porous BN structure in the inner.

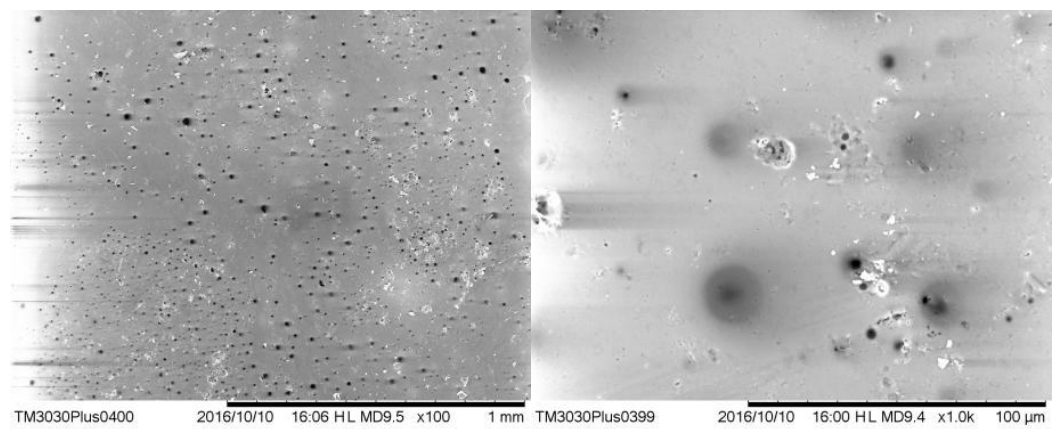

(a) $\times 100$

(b) $\times 1000$

Fig.6 .2 the compact $\mathrm{B}_{2} \mathrm{O}_{3}$ layer on the surface.

\section{Conclusions}

Boron nitride intumescent fire retardant coating containing borax and melamine as the main components was experimentally studied. This novel B-N intumescent fire retardant coating is completely different form the P-C-N coating in intumescent and heat resistance mechanism. No char layers are formed. The surface of coating forms a layer of dark green, compact glazed $\mathrm{B}_{2} \mathrm{O}_{3}$ while the internal structure of coating forms porous spongy BN. This structure makes the coating has a high heat resistance. On the other hand, because of the low melting point of borax the coating can't withstand high temperature; it will soften under high temperature. This has seriously affected 
the fire resistance time of the coating. Method for solving this problem is to find a new kind of nitrogen containing compounds that can make the reaction of borax to BN more easily. Further research on this aspect should be continued as the number of nitrogen containing compounds is numerous. The experiments in this paper prove that intumescent fire retardant coating with borax and nitrogen containing compound is feasible.

\section{References}

[1] GB14907-2002, Fire Resistive Coating for Steel Structure [S]. In Chinese.

[2] Jun-wei Gu and Guang-cheng Zhang and et al, Study on preparation and fire-retardant mechanism analysis of intumescent flame-retardant coatings [J]. Surface and Coatings Technology, 2007, 201(18): 7835-7841.

[3] Ji Wang and Wen-hua Song and Miao Zhang and et al, Experimental Study of the Acid Corrosion Effects on an Intumescent Coating for Steel Elements [J]. Industrial \& Engineering Chemistry Research, 2014, 53: 11249-11258.

[4] Yanchao Shi and Guojian Wang, The novel silicon-containing epoxy/PEPA phosphate flame retardant for transparent intumescent fire resistant coating [J]. Applied Surface Science, 2016.11, 385: 453-463.

[5] Yanchao Shi and Guojian Wang, The novel epoxy/PEPA phosphate flame retardants: Synthesis, characterization and application in transparent intumescent fire resistant coatings [J]. Progress in Organic Coatings, 2016.8, 97: 1-9.

[6] Yanchao Shi and Guojian Wang, An intumescent flame retardant containing caged bicyclic phosphate and oligomer: Synthesis, thermal properties and application in intumescent fire resistant coating [J]. Progress in Organic Coatings, 2016.1, 90: 83-90.

[7] Guojian Wang and Yan Huang and Xiang Hu, Synthesis of a novel phosphorus-containing polymer and its application in amino intumescent fire resistant coating [J]. Progress in Organic Coatings, 2013.1, 76(1): 188-193.

[8] Xiong Jiajin and Qiu Ludao and Shi Hua and et al, Intumescent Fire Retardant Coating Testing Furnace [P]. Chinese patent, CN1975371A, 2006-12-21. In Chinese.

[9] GB/T 9978.1-2008, Fire resistance tests-Elements of building construction-Part1: General requirements $[\mathrm{S}]$. In Chinese. 